$\underline{\text { Research article }}$

\title{
Uric acid lowering treatment alleviates perivascular carotid collar placement induced neointimal lesions in Uricase knockout mice
}

\author{
Running title: Hyperuricemia contributes to neointimal lesions \\ Jie Lu ${ }^{1,2,3^{*}}$, Ming-Shu Sun ${ }^{1,2,4^{*}}$, Xin-Jiang $\mathrm{Wu}^{1 *}$, Xuan Yuan ${ }^{1}$,Zhen $\mathrm{Liu}^{1,2}$, Xiao-Jie $\mathrm{Qu}^{1,2,3}$, Xiao-Peng Ji ${ }^{1,2}$, \\ Tony R Merriman ${ }^{5}$, Chang-Gui $\mathrm{Li}^{1,2,3}$ \\ ${ }^{1}$ Institute of Metabolic Diseases, Qingdao University, Qingdao, China; ${ }^{2}$ Shandong Provincial Key \\ Laboratory of Metabolic Diseases and Qingdao Key Laboratory of Gout, the Affiliated Hospital of \\ Qingdao University, Qingdao, China; ${ }^{3}$ Department of Endocrinology and Metabolic Diseases, the \\ Affiliated Hospital of Qingdao University, Qingdao, China; ${ }^{4}$ Department of Rheumatology and Clinical \\ Immunology, the Affiliated Hospital of Qingdao University, Qingdao, China; ${ }^{5}$ Department of \\ Biochemistry, University of Otago, Dunedin, New Zealand. \\ * These authors contributed equally to this article.
}

Correspondence to: Changgui Li, Shandong Provincial Key Laboratory of Metabolic Diseases, 16 Jiangsu Road, the Affiliated Hospital of Qingdao University, Qingdao 266003, China. Email: changguili@vip.163.com or Tony R Merriman, Department of Biochemistry, 710 Cumberland Street, University of Otago, Dunedin 9016, New Zealand. Email:tony.merriman@otago.ac.nz.

Keywords: urate, atherosclerosis, reactive oxygen species, allopurinol, animal model

Summary statement: We generated a carotid collar placement atherosclerosis model in the novel spontaneous HU Uox-KO mouse and demonstrate that urate plays a contributing rather than a causal role in the carotid neointimal lesions, while urate-lowering treatment may bring additional benefits in this HU mouse model. 


\begin{abstract}
Hyperuricemia (HU) is a cause of gout. Clinical studies show a link between HU and cardiovascular disease. However, the role of soluble serum urate on atherosclerosis development remains elusive. We aimed to use a new HU mouse model (Uricase/Uox knockout $(\mathrm{KO})$ ) to further investigate the relationship between $\mathrm{HU}$ and atherosclerosis. Mouse model of induced carotid atherosclerosis was established in the novel spontaneous HU Uox-KO mouse and their wild type littermates (C57BL/6J background). Mice were implanted with a perivascular collar placement around the right carotid artery in combination with a western-type diet. To investigate urate-lowering treatment (ULT) effects on intima, the mice were gavaged daily from the age of 6 weeks with allopurinol. Human umbilical vein endothelial cells (HUVECs) were co-incubated with soluble urate, with and without probenecid, to study the mechanism of urate-related atherosclerosis. The Uox-KO mice had significantly elevated serum urate levels combined with higher blood urea nitrogen and serum creatinine. Western blot analysis showed enhanced levels of atherosclerosis inflammatory response proteins. However, there were no other risk indicators for the pathogenesis of atherosclerosis, including increased fasting glucose, altered lipid and atherosclerosis characterized cardiovascular and histological manifestations. In contrast, collar placement Uox-KO mice showed severe neointimal changes in histology staining consistent with increases in intimal area and increases in proliferating cell nuclear antigen (PCNA) - and F4/80-positive cells. Allopurinol reduced neointimal areas induced by the perivascular collar in hyperuricemic mice accompanied by decreased expression of PCNAand F4/80-positive cells $(P<0.05)$. ULT alleviated atherosclerosis inflammatory response factors and reactive oxygen species intensities in both collar placement Uox-KO mice and urate-stimulated HUVECs. In vitro results using HUVECs showed ROS was induced by urate and ROS induction was abrogated using antioxidants. These data demonstrate that urate per se does not trigger atherosclerosis intima lesions in mice. Urate worsens carotid neointimal lesions induced by the perivascular collar and urate-lowering therapy partially abrogates the effects. The current study warrants the further human based study on the possible benefits of urate-lowering therapy in atherosclerosis patients with HU.
\end{abstract}




\section{Introduction}

Cardiovascular disease (CVD) is the leading cause of death world-wide (Lozano et al., 2012). Atherosclerosis is one of the major CVDs characterized by focal intimal thickening and ultimately luminal obstruction induced by fibro-proliferation and an inflammatory process mediated by cytokine production and vascular regulatory mechanisms (Zmuda et al., 2011). Neointimal formation happens at the early stage of atherosclerosis which is a complex process initiated by the damage of endothelial cells and exposure of vascular smooth muscle cells to circulating blood elements (Tian et al., 2017). Hyperuricemia (HU) causes gout and has been implicated in hypertension and atherosclerosis in humans (Odden et al., 2014). Epidemiological studies have associated HU with atherosclerotic vascular diseases (Wu et al., 2016), and the level of serum urate (SU) can predict cardiovascular outcomes and mortalities in both sexes (Xia et al., 2014). HU is also a risk factor for hypertension, which is a potent contributor of atherosclerosis ( $\mathrm{Wu}$ and Chan, 2012). A meta-analysis of 18 prospective cohort studies, including data from more than 55,000 patients, showed an increased risk of incident hypertension in subjects with $\mathrm{HU}$, and the overall risk increased by $13 \%$ per 1 $\mathrm{mg} / \mathrm{dL}$ increase in SU (Grayson et al., 2011). More recently, a retrospective cohort study demonstrated that HU plays a significant role in the progression from prehypertension to hypertension with a 35\% increasing ratio of hypertension risk in men (Do et al., 2015). These findings have prompted a growing research interest on the possible benefits of urate-lowering treatment (ULT) in cardiovascular diseases. However, it has not been definitively established whether urate is merely a marker or a causal agent of CVD, or whether ULT affects outcomes. This study was designed to investigate the relationship between urate and atherosclerosis in a spontaneous HU mouse model with collar-induced neointimal lesions.

\section{Results}

\section{Soluble urate does not induce atherosclerotic phenotypes}

SU levels in Uox-KO mice were almost 3-fold increased compared to WT counterparts $(563.9 \mu \mathrm{mol} / \mathrm{L} \pm 16.9$ vs. $176.3 \mu \mathrm{mol} / \mathrm{L} \pm 6.7, P<0.001$; Table 1). Compared with WT controls, BUN and serum creatinine were significantly elevated in Uox-KO mice (Table 1). No apparent changes occurred in the fasting glucose level of Uox-KO mice compared with WT mice (Table 1). Neither did lipid profiles change (TC, HDL-C and LDL-C; Table 1).

To evaluate the effects of urate on the cardiovascular system, blood pressure, endothelium-dependent vasodilatation and cardiac function were tested. Systolic blood pressure (SBP), diastolic blood pressure (DBP) and mean blood pressure (MBP) exhibited no 
difference between Uox-KO mice and WT controls (Fig. 1A). Neither did aortae dilation nor cardiovascular function change (Fig. 1B; Table 2). This does not support a direct role of urate in the control of blood pressure, vasodilation and cardiac function. As shown in Fig 1C and $1 \mathrm{D}$, pathogenic proteins in atherosclerosis were up-regulated in both plasma and carotid lesions - MCP-1, ICAM-1, and VCAM-1 ( $P<0.05)$. However, Uox-KO mice did not present an atherosclerotic phenotype, showing no changes in intimal areas, proliferation (proxied by PCNA- positive cells) and inflammation (proxied by F4/80-positive cells) in Fig. 1E and 1F.

\section{Hyperuricemia accelerates carotid neointimal lesions with collar placement}

We next addressed the questions: is the effect of urate evident only once a stress is given, and does ULT affect the atherosclerosis pathogenesis? This perivascular carotid collar model along with western-type diet represents a useful complementary model in which neointimal lesions show up at the early-stage of atherosclerosis. With collar induction HU makes neointimal lesions worse, indicated by the increases in intimal area, more PCNA- and F4/80positive cells in the carotid artery compared with WT collared mice (Fig. 2C, 2D). Administration of allopurinol $(100 \mathrm{mg} / \mathrm{kg})$, a xanthine oxidase inhibitor and urate-lowering drug, significantly alleviated the intimal phenotypes including intimal area, PCNA-positive cell counts and F4/80 positive cell counts. Allopurinol also alleviated both SU and renal functions indicated by BUN and creatinine in $U o x-\mathrm{KO}$ collared mice compared to WT collared mice (Table 1). Consistently, allopurinol decreased expression of MCP-1, as well as ICAM-1 and VCAM-1, in both plasma and carotid tissues determined by ELISA and RTPCR, respectively (Fig. 2A, 2B). The down-regulation of MCP-1, ICAM-1, and VCAM-1 in carotid tissue indicated that inflammation was improved by ULT. Echocardiographic analysis of heart rate, diastolic left ventricular internal diameter and ejection fraction exhibited no difference between collared Uox-KO mice and WT controls (data not shown).

\section{Hyperuricemia elevates ROS in carotid artery in vivo}

Given ROS plays a putative role in the pathogenesis of atherosclerosis, we tested the ROS intensity by fluorescent dye dihydroethidium staining in the carotid artery of WT and UoxKO mice. Uox-KO mice had elevated ROS levels compared with WT, with or without collar placement (Fig 3A, 3B). ROS intensities were reduced in Uox-KO mice with $100 \mathrm{mg} / \mathrm{kg}$ allopurinol treatment for 10 weeks versus WT controls in the presence or absence of collar placement (Fig 3A, 3B), implying that urate imposes additional oxidative stress which may accelerate atherosclerosis development. 


\section{Soluble urate induces ROS enhancement in vitro}

Human umbilical vein endothelial cell (HUVEC) $\left(10^{5} /\right.$ well) viability was measured when coincubated with soluble urate $(200 \mu \mathrm{mol} / \mathrm{L}, 400 \mu \mathrm{mol} / \mathrm{L}, 600 \mu \mathrm{mol} / \mathrm{L}$ and $800 \mu \mathrm{mol} / \mathrm{L})$ at $24 \mathrm{~h}$, $48 \mathrm{~h}$ and $72 \mathrm{~h}$. As shown in Fig.4A to $4 \mathrm{C}$, soluble urate decreased cell viability in a dosedependent and time-dependent manner and elevated ROS levels - for example with 800 $\mu \mathrm{mol} / \mathrm{L}$ urate, ROS intensity was almost 10-fold increased. ROS levels were extenuated by pre-incubation for $12 \mathrm{~h}$ with probenecid $(1 \mathrm{mmol} / \mathrm{L})$, an organic anion transport inhibitor, or $\mathrm{N}$-acetylcysteine (NAC) $(10 \square \mu \mathrm{mol} / \mathrm{L})$, a ROS scavenger, or with both (Fig. 4D). Relative mRNA expression of MCP-1, ICAM-1, and VCAM-1 in soluble urate (800 $\mu \mathrm{mol} / \mathrm{L}, 48 \mathrm{~h})$ treated HUVECs was significantly higher than controls and this was lessened by preincubation with probenecid or NAC or both (Fig. 4E).

\section{Discussion}

The presence of hepatic Uox is the reason that rodents have lower SU compared to humans (Alvarez-Lario and Macarron-Vicente, 2010). When Uox is knocked out, mice develop spontaneous HU similar to the SU level of humans (Lu et al., 2018). Based on this HU mouse model, we further generated an atherosclerosis model with right carotid artery peri-collar placement and a western-type diet. Using this model we showed that, because Uox-KO mice did not present an atherosclerotic phenotype despite of facts that inflammatory response and ROS indeed were induced in Uox-KO mice. However, HU worsened the development of neointimal lesions through in vivo ROS enhancement.

Epidemiological studies have detected an association between HU and hypertension, while evidence for causation, that includes data from Mendelian randomization studies, is limited and inconclusive (Dehghan et al., 2008; Sluijs et al., 2015; Vitart et al., 2008). Hypertension is a risk factor in the pathogenesis of atherosclerosis. Here we describe the cardiovascular characteristics in Uox-KO male mice, which exhibit no signs of heart dysfunction, heart morphology alteration or blood pressure change. This is consistent with data from another HU mouse model with liver Glut9 deficiency (Preitner et al., 2015). These data therefore do not support a direct causal role of urate on blood pressure in mice.

To our knowledge, this study is the first to assess conducted dilation responses of aortae in the spontaneous HU mouse and showed that urate cannot induce experimentally significant changes of endothelium-dependent vasodilatation. Indeed, when expressed as a function of the dilation, the conducted dilation of aortae in Uox-KO and WT mice was 
relatively similar. Interestingly, the endothelial relaxation dysfunction reported in aortae from low-density lipoprotein receptor/apolipoprotein E (LDL/ApoE) double knockout mice was in regions with significant lesions, and not in other regions or in aortae from the ApoE single KO mice where lesions were minimal (Beleznai et al., 2011). Similarly, even in diabetic ApoE-KO mice, endothelial dysfunction was only reported in plaque-prone regions of the aortae, while plaque-resistant segments maintained a normal acetylcholine response (Ding et al., 2005). These pieces of evidence are consistent with our observation of no vasodilation dysfunction in our Uox-KO mice compared with WT mice.

Our results show that spontaneous HU without any stress does not induce obvious atherosclerosis phenotypes. However, hyperuricemia alone was sufficient to induce oxidative stress and enhance levels of atherosclerosis associated inflammatory cytokines indicating that $\mathrm{HU}$ is only a promoting factor rather than an initiator in atherosclerosis. HU may contribute to an activated atherosclerotic pathological process. MCP-1, ICAM-1 and VCAM-1, crucial pathogenic elements in atherosclerosis, are up-regulated in atherosclerotic lesions and influence growth factor production and medial smooth muscle cell migration (Dzau et al., 2002; Hsueh et al., 2016). HU enhanced MCP-1, ICAM-1 and VCAM-1 as shown in serum level and protein expression, suggesting that these three molecules are also involved in a HUdriven atherosclerosis promoting effect that would exacerbate the pathogenesis of atherosclerosis. The augmented response of MCP-1, ICAM-1 and VCAM-1 to a given concentration of urate would stimulate downstream inflammation and thereby induce atherosclerosis progressing to a greater extent than controls in vitro. Increasing of intimal area due to smooth muscle cell movement and reproduction is an essential component of atherosclerosis, which can be indicated by proliferating cell nuclear antigen (PCNA) (Lu et al., 2017). Macrophages accumulate in atherosclerotic plaques, playing crucial roles in atherosclerotic immune responses ( $\mathrm{Li}$ et al., 2017). Significant increases in intimal areas, PCNA- and F4/80- positive cells in the carotid artery indicates that HU contributes to atherosclerosis, though no plague were found. Despite a few hundred systematic reviews, meta-analyses, and Mendelian randomisation studies exploring 136 unique health outcomes, convincing evidence of a clear causal role of urate in disease pathogenesis only exists for gout and nephrolithiasis (Liu et al., 2015). Urate is involved in a diverse array of biological functions, while possibly contributing to the pathogenesis of cardiovascular phenotypes, rendering it a pathogenic but not causal role (Alvarez-Lario and Macarron-Vicente, 2010). HU did associate with an increased risk of cardiovascular death only in participants with gout 
and existing cardiovascular disease (Muoio and Newgard, 2008), consistent with our experimental in vivo data.

Reactive oxygen species are another major but non-specific mediator in the formation of atherosclerosis-inducing endothelial dysfunction. It can reduce the bioavailability of nitric oxide, a potential anti-atherosclerotic factor (Montezano and Touyz, 2012). Urate can lead to ROS enhancement which facilitates atherosclerosis by oxidative stress (Forstermann et al., 2017), which is consistent with our in vivo and in vitro data. Changes in ROS exhibited a similar trend as the change of neointimal lesions in collar-induced Uox-KO mice, which were partially rescued by ULT. This suggests that ROS may also contribute to urate-induced atherosclerosis-promoting effects. The effect of allopurinol (via the active metabolite oxypurinol) that inhibits xanthine oxidase (XO) activity and suppresses urate biosynthesis (Elion, 1966), also reduces ROS production by inhibiting XO. Other non-XO effects of allopurinol include LDL oxidation prevention, heat shock protein expression inhibition and decreasing early changes in inflammation such as leukocyte activation by reducing adherence, rolling and extravasation (George and Struthers, 2009). Given our data shows HU was sufficient to induce both ROS production and atherosclerosis associated inflammatory cytokines, worsened ROS level, inflammatory molecules and neointimal lesions observed in collared Uox-KO mice could be effects due to an "additional action" imposed by HU. Thus, all the neointimal lesions and associated inflammatory factors as well as ROS production were alleviated when ULT was present. In vitro, our study showed the same trend of ROS in vivo. ROS intensities were lowered with probenecid intervention. Combined with probenecid and NAC, the strongest ROS lowering effect was observed (in HUVECs). Thus, the benefits of XO inhibitors such as allopurinol might rely on blocking the production of oxidants rather than on lowering urate (Johnson et al., 2013). A random, double-blind, crossover study also showed that the mechanism of improvement in endothelial function with high-dose allopurinol lies in its ability to reduce vascular oxidative stress and not in urate reduction (Civelek and Lusis, 2014). Therefore, although our data exclude a direct causal role of urate per se on atherosclerosis in Uox-KO mice, further studies to address a potential role of $\mathrm{XO}$ activity on the cardiovascular function are warranted. Overall, this work reinforces the conclusion that urate accelerates pathogenesis of atherosclerosis and ROS lowering may bring the anti-atherosclerotic effects.

It remains controversial as to whether asymptomatic HU should be treated for the purpose of improving cardiovascular outcomes (Abeles, 2015). Stamp and Dalbeth (Stamp and Dalbeth, 2017) suggested that asymptomatic HU treatment needs to be cautiously 
considered, due to the limited data and the potential risks of treatment. Kok et al. (Kok et al., 2014) reported that allopurinol therapy in patients with gout does not yield beneficial cardiovascular outcomes. However, Kuwabara et al. (Kuwabara et al., 2017) promoted the use of ULT for asymptomatic HU in a five-year Japanese cohort study with 13,201 subjects. ULT was associated to better outcomes in HU patients with cardiovascular diseases accompanied by benefits in endothelial dysfunction and systemic inflammation (Hayashino et al., 2016). A randomised controlled trial reported that allopurinol reduces central blood pressure and carotid intima-media thickness progression at 1 year in patients with recent ischemic stroke and transient ischemic attack (Higgins et al., 2014). A mouse study reported that allopurinol represents a potential novel strategy for preventing left ventricular remodelling and dysfunction after myocardial infarction (Engberding et al., 2004). As well, significant anti-atherosclerotic effects were seen in a HU study in ApoE-KO mice (Wakuda et al., 2014). Consistently, our results suggest that ULT with allopurinol for hyperuricemic mice may improve neointimal lesions significantly.

Uox-KO mice did not show significant difference in blood pressure, vasodilation or atherosclerotic lesions as compared with controls even urate concentration $>560 \mu \mathrm{mol} / \mathrm{L}$, comparable to human (Feig et al., 2008). Even though the Uox-KO mice developed chronic renal dysfunction with a trend for increased plasma BUN, creatinine and chronic renal inflammation and fibrosis ( $\mathrm{Lu}$ et al., 2018), there was no correlation between renal or metabolic dysfunction and atherosclerosis development in this mouse model.

It is important to note that there was only approximately $40 \%$ of survivors in the UoxKO birth cohort (Lu et al., 2018). Thus, the survived Uox-KO mice with constant high UA levels may represent biased subjects and they normally develop significant metabolic and renal dysfunction, which actually mimics the human cases of gout and hyperuricemia with severe complications. A transient increase in uricosuria during the first two weeks of life in Uox-deficient mice might be responsible for the relative low survival rate (Flannick and Florez, 2016). The current model is useful to study the asymptomatic HU from a metabolic perspective though it is not fully understood the reasons of poor survival rate.

There is phenotypic heterogeneity existed between sexes in the Uox-KO model. Female Uox-KO mice have elevation in blood pressure compared with WT counterparts, while males do not (Lu et al., 2018). In addition, the association between SU and hypertension might vary according to age and sex, being more significant in younger and female subjects (Batzoglou and Schwartz, 2014). To avoid confounding factor due to gender or sex hormone difference, 
we only chose male mice in the current study. Further investigation in females would help to delineate a full picture of the effect of HU on cardiovascular diseases.

In conclusion, this is the first evidence to demonstrate that urate plays a contributing rather than a causal role in the carotid neointimal lesions, while ULT may bring additional benefits in this spontaneous HU mouse model. Clinical and mechanism studies are warranted to investigate the ULT's anti-atherosclerotic benefits in atherosclerosis patients with HU.

\section{Materials and Methods}

\section{Mouse model}

The spontaneous HU mice were developed by knock-out of the hepatically-expressed Uricase (Uox) gene (Lu et al., 2018). Controls were their wild type (WT) littermates on the C57BL/6J background. As previously described (Baetta et al., 2007), we generated perivascular carotid collar placement mouse models with non-occlusive silastic collars (length, $5 \mathrm{~mm}$; internal diameter, $0.3 \mathrm{~mm}$; external diameter, $0.6 \mathrm{~mm}$ ) in 10-week-old males. Four weeks before surgery, the animals received high-fat, high-cholesterol (HF/HC) westerntype diets $(21 \%(\mathrm{wt} / \mathrm{wt})$ fat and $0.15 \%$ cholesterol). Mice were anesthetized by ketamine/xylazine and kept on continuous anesthesia during the surgery. Subsequently, a midline neck incision was made to surgically expose the right carotid artery, and the collar was positioned around the right carotid artery and held in place with a nylon sleeve. Both carotid sheaths were openned, and the common carotid arteries were dissected free from the surrounding connective tissue. The carotid arteries were then returned to their original position and the wound was sutured. After a 6-week collar placement with western-type diet, all mice were sacrificed for subsequent measurements. To evaluate the urate-lowering effect, mice were gavaged with allopurinol at a dose of $100 \mathrm{mg} / \mathrm{kg}$ from 6 weeks to 16 weeks of age with collar induction at 10-week-old.

Mice were housed under specific pathogen free (SPF) conditions at $22^{\circ} \mathrm{C}$ under a $12-\mathrm{h}$ light/dark photoperiod with ad libitum access to rodent diet and sterile water (Zhang et al., 2010). The Animal Research Ethics Committee of the Affiliated Hospital of Qingdao University approved this study.

\section{Blood biochemistry}

Mice were fasted overnight before blood collection from the outer canthus. All biochemical indicators including SU, blood urea nitrogen (BUN), serum creatinine, fasting glucose, lipid 
profiles (total cholesterol (TC), triglyceride (TG), high- and low- density lipoprotein cholesterol (HDL-C, LDL-C) were measured by an automatic biochemical analyser (Toshiba).

\section{ELISA}

Plasma monocyte chemoattractant protein-1 (MCP-1), intercellular adhesion molecule-1 (ICAM-1) and vascular cell adhesion molecule-1 (VCAM-1) levels were quantified using an enzyme-linked immunosorbent assay (ELISA) kit (R\&D Systems).

\section{Quantitative real-time $R T$-PCR}

Total RNA was isolated from the carotid artery or cell line using trizol reagent (Roche Pharmaceuticals) and then reverse transcribed using a Fast Quant RT kit (Takara-Bio), followed by amplification using primers for MCP-1, ICAM-1 and VCAM-1 under the condition: $95^{\circ} \mathrm{C}$ for $10 \mathrm{~min}$ and 40 cycles of $95^{\circ} \mathrm{C}$ for $15 \mathrm{~s}, 58^{\circ} \mathrm{C}$ for $20 \mathrm{~s}$ and $68^{\circ} \mathrm{C}$ for $20 \mathrm{~s}$. The threshold cycle $(\mathrm{Ct})$ was determined and used to calculate $\Delta \mathrm{CT}$ values. The $\Delta \Delta \mathrm{Ct}\left(2^{-\Delta \mathrm{Ct}}\right)$ was used to calculate relative mRNA expression, with each measurement performed in triplicate. Primer sequences are shown in Supplementary Table 1.

\section{Western blot}

Protein was extracted from the carotid artery. $50 \mu \mathrm{g}$ protein lysates were loaded and transferred to nitrocellulose membranes. Blots were incubated with the primary antibodies (1:2000, Abcam) against MCP-1 (Catalog no. ab25124), ICAM-1 (Catalog no. ab119871), VCAM-1 (Catalog no. ab134047) and GAPDH (Catalog no. ab8245) at $4^{\circ} \mathrm{C}$ overnight. After incubation with horseradish peroxidase-conjugated goat anti-rabbit secondary antibodies, visualization was performed with an enhanced chemiluminescence kit (Thermo Scientific). Each measurement was performed in triplicate.

\section{Echocardiographic analysis}

Echocardiography was performed after anesthesia with isoflurane using a Vevo 2100 ultrasound system equipped with a MS400 probe (VisualSonics) for in vivo transthoracic ultrasound imaging. The heart was imaged in a 2-dimensional mode in the parasternal longaxis view. An M-mode cursor was positioned perpendicular to the interventricular septum and the posterior wall of the left ventricle at the level of the papillary muscles. Stroke volume 
was calculated as (LV Vol; $\mathrm{d}-\mathrm{LV}$ Vol; s) and cardiac output as ([LV Vol; d - LV Vol; s] $\times$ HR) / 1000.

\section{Blood pressure}

Systolic (SBP) and diastolic (DBP) blood pressure was measured by the CODA programmable noninvasive tail-cuff sphygmomanometer (Kent Scientific). Mice underwent an acclimation period of 7 consecutive days to the sphygmomanometer before experiments. Mean blood pressure (MBP) was calculated as (DBP + 1/3 [SBP - DBP]).

\section{Assessment of endothelium-dependent vasodilatation}

Vasorelaxation of isolated aortic ring segments were determined in oxygenated Kreb's solution. Aortic rings were precontracted with $0.1 \mu \mathrm{mol} / \mathrm{L}$ noradrenaline after an equilibration period of $60 \mathrm{~min}$. Dilation at each acetylcholine $(0.001,0.01,0.1,1.0,10 \mu \mathrm{mol} / \mathrm{L})$ concentration was measured and expressed as the percentage in response to noradrenaline.

\section{Pathology analysis and immunohistochemistry}

Carotid arteries were removed and fixed in formalin followed by paraffin embedding of $5 \mu \mathrm{m}$ serial sections. Tissue serial sections were incubated with anti-mouse proliferating cell nuclear antigen (PCNA, 1:50, Santa Cruz, Catalog no. sc25280) and anti-mouse F4/80 (macrophage-specific marker, 1:50, Santa Cruz, Catalog no. sc52664) rabbit polyclonal antibodies. Images were captured by Nikon Eclipse TE2000-S microscope (Nikon) and analysed by Image-Pro Plus software (version 6.0).

\section{Reactive oxygen species (ROS) measurement}

Samples were incubated with $2 \mu \mathrm{mol} / \mathrm{L}$ dihydroethidium (DHE) fluorescence probe (Thermo Scientific) for $30 \mathrm{~min}$ at $37^{\circ} \mathrm{C}$ in the dark to measure ROS levels. Fluorescence was determined using a Nikon 90i (Nikon) with excitation wavelength at $480 \mathrm{~nm}$ and emission wavelength at $610 \mathrm{~nm}$.

\section{Soluble urate}

As previously described (Eisenbacher et al., 2014), soluble urate was prepared by dissolving uric acid (UA, Sigma) in warmed media containing $1 \mathrm{M} \mathrm{NaOH}$. The solution was tested to be 
free of mycoplasma, endotoxin and filtered before use. Crystals were not detectable under polarizing microscopy, nor did they develop during cell incubation.

\section{Cell Culture}

Human umbilical vein endothelial cells (HUVECs, Cell bank of Chinese Academy of Sciences) were cultured in human endothelial cell-specific growth medium C-22010 (PromoCell) with 10\% fetal bovine serum, 100 units $/ \mathrm{ml}$ penicillin and $100 \mu \mathrm{g} / \mathrm{ml}$ streptomycin (Invitrogen). The viability of HUVECs was measured by Cell Counting Kit-8 (Beyotime Institute of Biotechnology) according to the manufacturer's instruction. Cells $\left(10^{5} /\right.$ well) were plated in 96 -well plates and co-incubated with soluble urate $(200-800 \mu \mathrm{mol} / \mathrm{L})$ after $1 \mathrm{mmol} / \mathrm{L}$ probenecid (an organic anion transport inhibitor that blocks UA entry into cells) or $10 \square \mu \mathrm{mol} / \mathrm{L} \mathrm{N}$-acetyl-1-cysteine (NAC, a ROS scavenger) intervention for 12 hours.

\section{Statistical analysis}

All statistical analyses were performed using GraphPad Prism software (version 7). Data were presented as the mean \pm SEM. Differences between groups were analyzed by Student's t test or one-way analysis of variance followed by Newman-Keuls multiple comparison test as appropriate. $P<0.05$ was considered to be statistically significant.

Acknowledgments All authors have written and edited the article.

Competing interests The authors declare no competing interests.

Funding This study is supported by the research project grants from National Key Research and Development Program (\#2016YFC0903400), National Science Foundation of China (\#81520108007, \#81770869, \#31371272, \#81500346, \#81441401, \#31471195), Science and Technology Development Project of Shandong Province (\#2014GSF118013), Basic Application Research Plan of Qingdao (\#15-9-1-98-jch).

\section{References}

Abeles, A. M. (2015). Hyperuricemia, gout, and cardiovascular disease: an update. Curr Rheumatol Rep 17, 13.

Alvarez-Lario, B. and Macarron-Vicente, J. (2010). Uric acid and evolution. Rheumatology (Oxford) 49, 2010-5. 
Baetta, R., Silva, F., Comparato, C., Uzzo, M., Eberini, I., Bellosta, S., Donetti, E. and Corsini, A. (2007). Perivascular carotid collar placement induces neointima formation and outward arterial remodeling in mice independent of apolipoprotein E deficiency or Western-type diet feeding. Atherosclerosis 195, e112-24.

Batzoglou, S. and Schwartz, R. (2014). Computational biology and bioinformatics. Bioinformatics 30, i1-2.

Beleznai, T., Takano, H., Hamill, C., Yarova, P., Douglas, G., Channon, K. and Dora, K. (2011). Enhanced K(+)-channel-mediated endothelium-dependent local and conducted dilation of small mesenteric arteries from ApoE(-/-) mice. Cardiovasc Res 92, 199-208.

Civelek, M. and Lusis, A. J. (2014). Systems genetics approaches to understand complex traits. Nat Rev Genet 15, 34-48.

Dehghan, A., Kottgen, A., Yang, Q., Hwang, S. J., Kao, W. L., Rivadeneira, F., Boerwinkle, E., Levy, D., Hofman, A., Astor, B. C. et al. (2008). Association of three genetic loci with uric acid concentration and risk of gout: a genome-wide association study. Lancet 372, 1953-61.

Ding, H., Hashem, M., Wiehler, W. B., Lau, W., Martin, J., Reid, J. and Triggle, C. (2005). Endothelial dysfunction in the streptozotocin-induced diabetic apoE-deficient mouse. Br J Pharmacol 146, 1110-8.

Do, O. H., Low, J. T. and Thorn, P. (2015). Lepr(db) mouse model of type 2 diabetes: pancreatic islet isolation and live-cell 2-photon imaging of intact islets. $J$ Vis Exp, e52632.

Dzau, V. J., Braun-Dullaeus, R. C. and Sedding, D. G. (2002). Vascular proliferation and atherosclerosis: new perspectives and therapeutic strategies. Nat Med $\mathbf{8}$, 1249-56.

Eisenbacher, J. L., Schrezenmeier, H., Jahrsdorfer, B., Kaltenmeier, C., Rojewski, M. T., Yildiz, T., Beyer, T., Erle, A., Wiegmann, D. S., Grassl, S. et al. (2014). S100A4 and uric acid promote mesenchymal stromal cell induction of IL-10+/IDO+ lymphocytes. J Immunol 192, 6102-10.

Elion, G. B. (1966). Enzymatic and metabolic studies with allopurinol. Ann Rheum Dis 25, 608-14.

Engberding, N., Spiekermann, S., Schaefer, A., Heineke, A., Wiencke, A., Muller, M., Fuchs, M., Hilfiker-Kleiner, D., Hornig, B., Drexler, H. et al. (2004). Allopurinol attenuates left ventricular remodeling and dysfunction after experimental myocardial infarction: a new action for an old drug? Circulation 110, 2175-9.

Feig, D. I., Kang, D. H. and Johnson, R. J. (2008). Uric acid and cardiovascular risk. $N$ Engl J Med 359, 1811-21.

Flannick, J. and Florez, J. C. (2016). Type 2 diabetes: genetic data sharing to advance complex disease research. Nat Rev Genet 17, 535-49.

Forstermann, U., Xia, N. and Li, H. (2017). Roles of Vascular Oxidative Stress and Nitric Oxide in the Pathogenesis of Atherosclerosis. Circ Res 120, 713-735.

George, J. and Struthers, A. D. (2009). Role of urate, xanthine oxidase and the effects of allopurinol in vascular oxidative stress. Vasc Health Risk Manag 5, 265-72.

Grayson, P. C., Kim, S. Y., LaValley, M. and Choi, H. K. (2011). Hyperuricemia and incident hypertension: a systematic review and meta-analysis. Arthritis Care Res (Hoboken) 63, 102-10.

Hayashino, Y., Okamura, S., Tsujii, S. and Ishii, H. (2016). Association of serum uric acid levels with the risk of development or progression of albuminuria among Japanese patients with type 2 diabetes: a prospective cohort study [Diabetes Distress and Care Registry at Tenri (DDCRT 10)]. Acta Diabetol 53, 599-607. 
Higgins, P., Walters, M. R., Murray, H. M., McArthur, K., McConnachie, A., Lees, K. R. and Dawson, J. (2014). Allopurinol reduces brachial and central blood pressure, and carotid intima-media thickness progression after ischaemic stroke and transient ischaemic attack: a randomised controlled trial. Heart 100, 1085-92.

Hsueh, T. P., Sheen, J. M., Pang, J. H., Bi, K. W., Huang, C. C., Wu, H. T. and Huang, S. T. (2016). The Anti-Atherosclerotic Effect of Naringin Is Associated with Reduced Expressions of Cell Adhesion Molecules and Chemokines through NF-kappaB Pathway. Molecules 21.

Johnson, R. J., Sanchez-Lozada, L. G., Mazzali, M., Feig, D. I., Kanbay, M. and Sautin, Y. Y. (2013). What are the key arguments against uric acid as a true risk factor for hypertension? Hypertension 61, 948-51.

Kok, V. C., Horng, J. T., Chang, W. S., Hong, Y. F. and Chang, T. H. (2014). Allopurinol therapy in gout patients does not associate with beneficial cardiovascular outcomes: a population-based matched-cohort study. PLoS One 9, e99102.

Kuwabara, M., Niwa, K., Hisatome, I., Nakagawa, T., Roncal-Jimenez, C. A., Andres-Hernando, A., Bjornstad, P., Jensen, T., Sato, Y., Milagres, T. et al. (2017). Asymptomatic Hyperuricemia Without Comorbidities Predicts Cardiometabolic Diseases: Five-Year Japanese Cohort Study. Hypertension 69, 1036-1044.

Li, Z., Zhou, Z., Hou, X., Lu, D., Yuan, X., Lu, J., Wang, C., Han, L., Cui, L., Liu, Z. et al. (2017). Replication of Gout/Urate Concentrations GWAS Susceptibility Loci Associated with Gout in a Han Chinese Population. Sci Rep 7, 4094.

Liu, R., Han, C., Wu, D., Xia, X., Gu, J., Guan, H., Shan, Z. and Teng, W. (2015). Prevalence of Hyperuricemia and Gout in Mainland China from 2000 to 2014: A Systematic Review and Meta-Analysis. Biomed Res Int 2015, 762820.

Lozano, R. Naghavi, M. Foreman, K. Lim, S. Shibuya, K. Aboyans, V. Abraham, J. Adair, T. Aggarwal, R. Ahn, S. Y. et al. (2012). Global and regional mortality from 235 causes of death for 20 age groups in 1990 and 2010: a systematic analysis for the Global Burden of Disease Study 2010. Lancet 380, 2095-128.

Lu, J., Hou, X., Yuan, X., Cui, L., Liu, Z., Li, X., Ma, L., Cheng, X., Xin, Y., Wang, C. et al. (2017). Knockout of the urate oxidase gene provides a stable mouse model of hyperuricemia associated with metabolic disorders. Kidney Int.

Lu, J., Hou, X., Yuan, X., Cui, L., Liu, Z., Li, X., Ma, L., Cheng, X., Xin, Y., Wang, C. et al. (2018). Knockout of the urate oxidase gene provides a stable mouse model of hyperuricemia associated with metabolic disorders. Kidney Int 93, 69-80.

Montezano, A. C. and Touyz, R. M. (2012). Reactive oxygen species and endothelial function--role of nitric oxide synthase uncoupling and Nox family nicotinamide adenine dinucleotide phosphate oxidases. Basic Clin Pharmacol Toxicol 110, 87-94.

Muoio, D. M. and Newgard, C. B. (2008). Mechanisms of disease:Molecular and metabolic mechanisms of insulin resistance and beta-cell failure in type 2 diabetes. Nat Rev Mol Cell Biol 9, 193-205.

Odden, M. C., Amadu, A. R., Smit, E., Lo, L. and Peralta, C. A. (2014). Uric acid levels, kidney function, and cardiovascular mortality in US adults: National Health and Nutrition Examination Survey (NHANES) 1988-1994 and 1999-2002. Am J Kidney Dis 64, 550-7.

Preitner, F., Pimentel, A., Metref, S., Berthonneche, C., Sarre, A., Moret, C., Rotman, S., Centeno, G., Firsov, D. and Thorens, B. (2015). No development of hypertension in the hyperuricemic liver-Glut9 knockout mouse. Kidney Int 87, 940-7.

Sluijs, I., Holmes, M. V., van der Schouw, Y. T., Beulens, J. W., Asselbergs, F. W., Huerta, J. M., Palmer, T. M., Arriola, L., Balkau, B., Barricarte, A. et al. (2015). A 
Mendelian Randomization Study of Circulating Uric Acid and Type 2 Diabetes. Diabetes 64, 3028-36.

Stamp, L. and Dalbeth, N. (2017). Urate-lowering therapy for asymptomatic hyperuricaemia: A need for caution. Semin Arthritis Rheum 46, 457-464.

Tian, D. Y., Jin, X. R., Zeng, X. and Wang, Y. (2017). Notch Signaling in Endothelial Cells: Is It the Therapeutic Target for Vascular Neointimal Hyperplasia? Int $J$ Mol Sci 18.

Vitart, V., Rudan, I., Hayward, C., Gray, N. K., Floyd, J., Palmer, C. N., Knott, S. A., Kolcic, I., Polasek, O., Graessler, J. et al. (2008). SLC2A9 is a newly identified urate transporter influencing serum urate concentration, urate excretion and gout. Nat Genet $\mathbf{4 0}$, 437-42.

Wakuda, H., Uchida, S., Ikeda, M., Tabuchi, M., Akahoshi, Y., Shinozuka, K. and Yamada, S. (2014). Is hyperuricemia a risk factor for arteriosclerosis? Uric acid and arteriosclerosis in apolipoprotein e-deficient mice. Biol Pharm Bull 37, 1866-71.

Wu, A. H., Gladden, J. D., Ahmed, M., Ahmed, A. and Filippatos, G. (2016). Relation of serum uric acid to cardiovascular disease. Int J Cardiol 213, 4-7.

Wu, M. and Chan, C. (2012). Learning transcriptional regulation on a genome scale: a theoretical analysis based on gene expression data. Brief Bioinform 13, 150-61.

Xia, X., He, F., Wu, X., Peng, F., Huang, F. and Yu, X. (2014). Relationship between serum uric acid and all-cause and cardiovascular mortality in patients treated with peritoneal dialysis. Am J Kidney Dis 64, 257-64.

Zhang, X., Zhu, X. and Chen, B. (2010). Inhibition of collar-induced carotid atherosclerosis by recombinant apoA-I cysteine mutants in apoE-deficient mice. $J$ Lipid Res 51, 3434-42.

Zmuda, E. J., Powell, C. A. and Hai, T. (2011). A method for murine islet isolation and subcapsular kidney transplantation. J Vis Exp. 


\section{Figure Legends}

Fig. 1. Soluble urate does not induce atherosclerosis phenotypes. A, Systolic (SBP), diastolic (DBP) and mean (MBP) blood pressure in non-fasting 10-week-old WT and Uox$\mathrm{KO}$ mice $(n=10,7)$. B, Endothelium-dependent vasodilatation of aortic segments in 10week-old WT and Uox-KO mice ( $n=3)$ induced by acetylcholine (Ach). C, Protein levels of MCP-1, ICAM-1, and VCAM-1 in serum of 10-week-old mice $(n=6)$ detected by ELISA. D, Protein levels of MCP-1, ICAM-1, and VCAM-1 in carotid arteries of 10-week-old mice determined by three independent western blotting experiments $(n=3)$. $\mathbf{E}$ and $\mathbf{F}$, Carotid pathological assessment in WT and Uox-KO mice $(n=6)$ quantified by intimal area, PCNAand F4/80- positive cell counts. Bars $=50 \mu \mathrm{m} .{ }^{*} P<0.05,{ }^{* *} P<0.01,{ }^{* * *} P<0.001$ versus WT. Error bars represent SEM.

Fig. 2. Hyperuricemia accelerates carotid neointimal lesions with collar placement. Shown are effects of collar induction and 10-week allopurinol treatment $(100 \mathrm{mg} / \mathrm{kg})$ on MCP-1, ICAM-1, and VCAM-1 levels in A plasma $(n=6)$ and $\mathbf{B}$ carotid tissues $(n=6)$, and carotid morphology, proliferation and inflammation represented by intimal area, PCNA- and F4/80- positive cell counts in $\mathbf{C}$ and $\mathbf{D}(n=6)$, respectively. Bars $=50 \mu \mathrm{m} .{ }^{* * *} P<0.001$ versus WT control and ${ }^{\dagger} P<0.05$ versus untreated $U o x$-KO mice. Error bars represent SEM.

Fig. 3. Hyperuricemia elevates ROS in carotid artery in vivo. A, Representative images of fluorescent dye dihydroethidium staining from carotid artery of WT and Uox-KO mice $(n=$ 6). B, Effects of 8-week allopurinol treatment $(100 \mathrm{mg} / \mathrm{kg})$ on ROS were quantified. Bars = $50 \mu \mathrm{m} .{ }^{* * *} P<0.001$ versus WT control. ${ }^{\dagger} P<0.05$ versus untreated $U o x$-KO mice. Error bars represent SEM.

Fig. 4. Soluble urate induces ROS enhancement in vitro. A, HUVECs (10 $/$ well) viability measurement co-incubated with soluble urate $(200,400$, and $800 \mu \mathrm{mol} / \mathrm{L})$ at $24 \mathrm{~h}, 48 \mathrm{~h}$ and 72h. B and C, Representative images of fluorescent dye dihydroethidium (DHE) staining from soluble urate stimulated HUVECs and quantified ROS levels. D, DHE staining on soluble urate $(800 \mu \mathrm{mol} / \mathrm{L}, 48 \mathrm{~h})$ stimulated HUVECs after $12 \mathrm{~h}$ pre-incubation with $1 \mathrm{mmol} / \mathrm{L}$ 
probenecid or $10 \square \mu \mathrm{mol} / \mathrm{L} \mathrm{N}$-acetyl-1-cysteine (NAC) or both. E, Relative mRNA expression of MCP-1, ICAM-1, and VCAM-1 in HUVECs. Data were represented as mean \pm SEM. Bars $=20 \mu \mathrm{m} .{ }^{*} P<0.05,{ }^{* *} P<0.01,{ }^{* * *} P<0.001$ versus control, ${ }^{\dagger} P<0.05$ versus UA group. Error bars represent SEM. 
Figure 1.

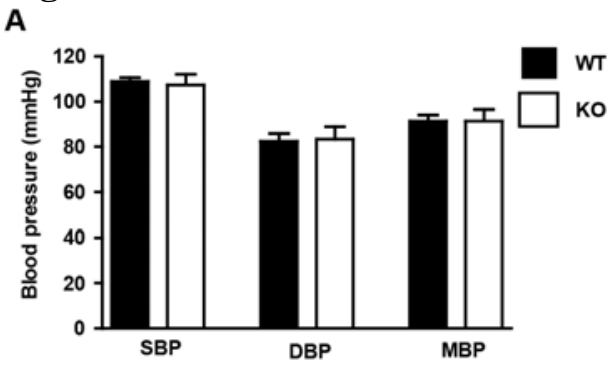

C
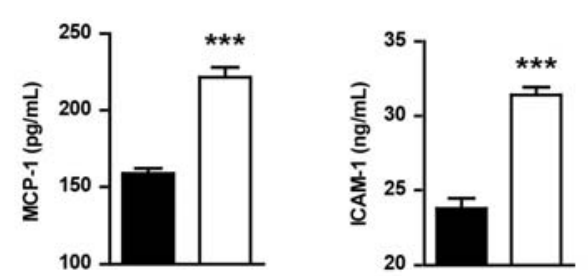

B
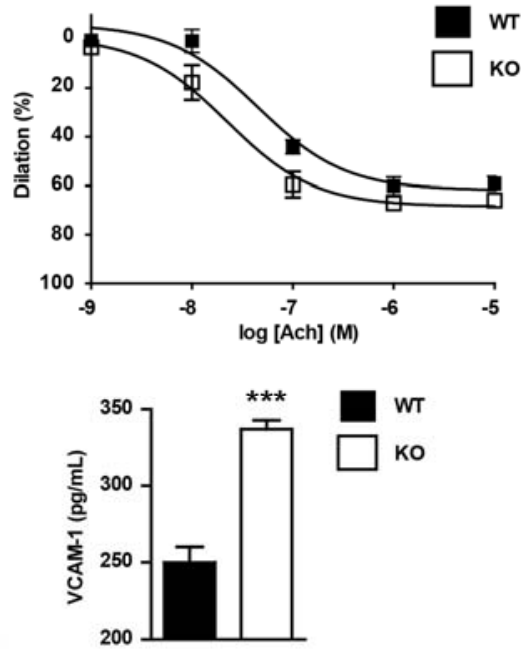

D
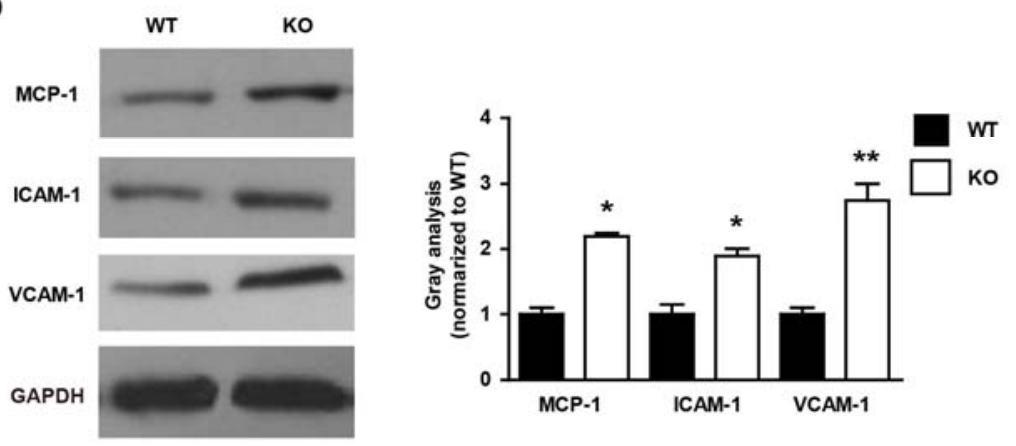

E
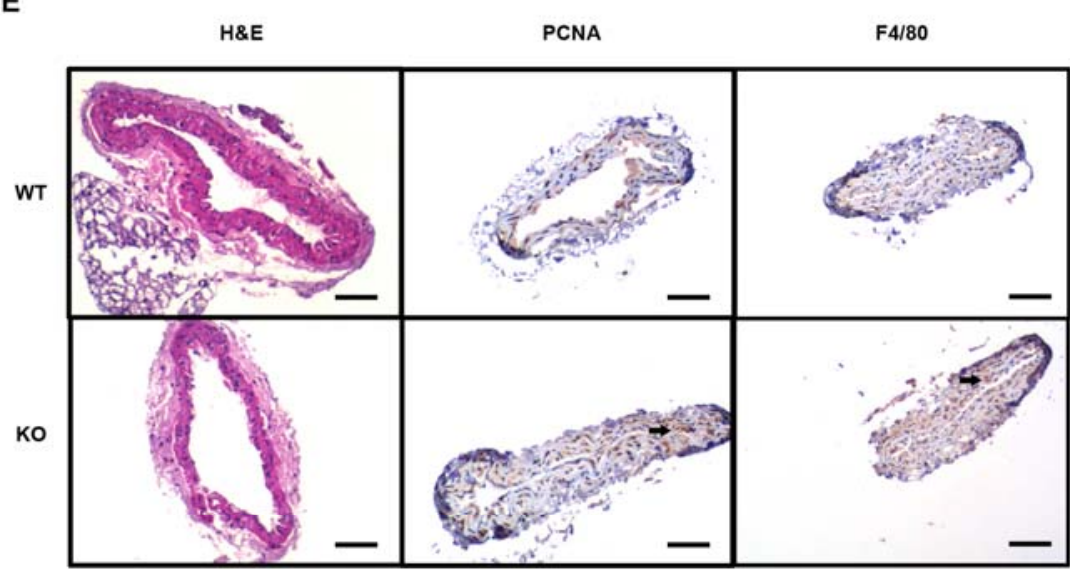

$\mathbf{F}$
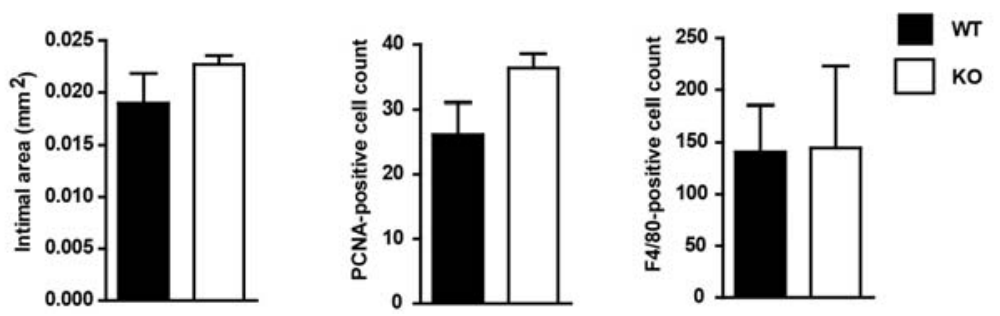
bioRxiv preprint doi: https://doi org/101101/366096; this version posted July 10 2018. The copyright holder for this preprint (which was not certified by peer review) is the author/funder, who has granted bioRxiv a license to display the preprint in perpetuity. It is made available under aCC-BY-NC-ND 4.0 International license.

Figure 2.

A
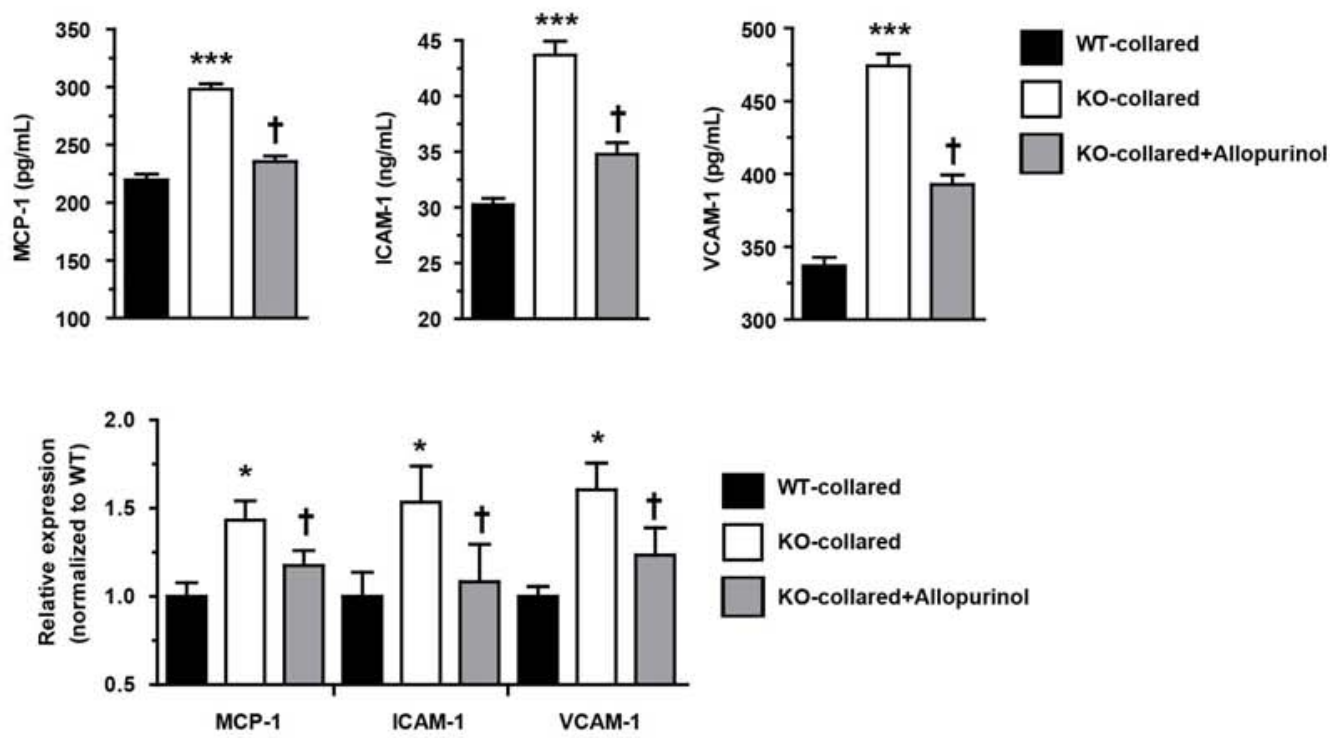

C

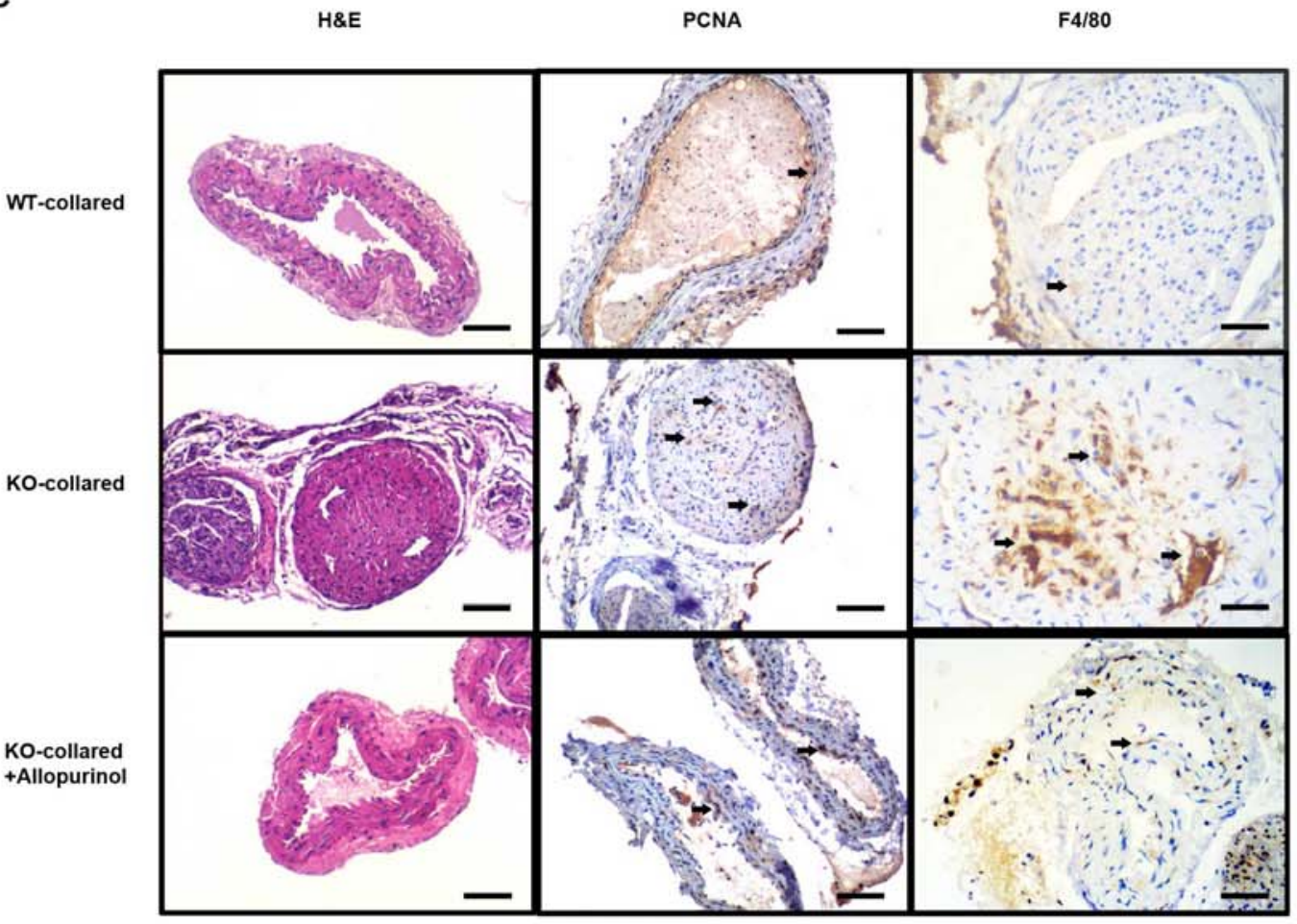

D
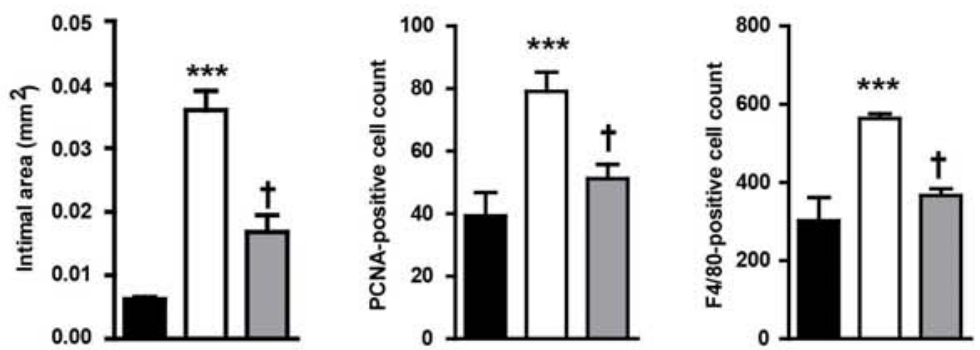

WT-collared

$\square$ ko-collared

ko-collared+Allopurinol 


\section{Figure 3.}

A
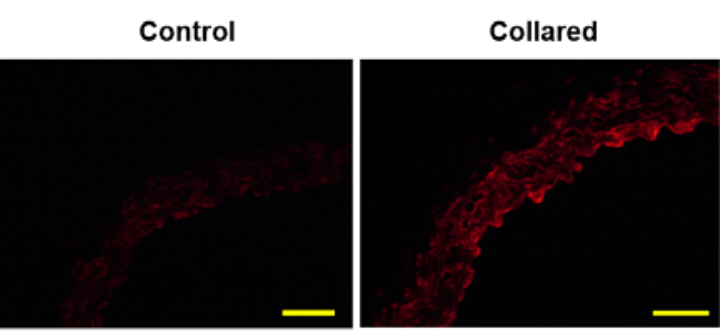

ko
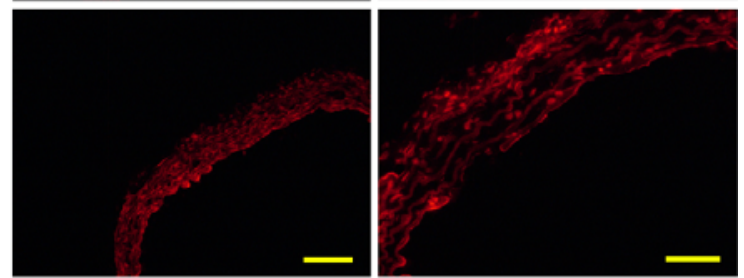

Ko
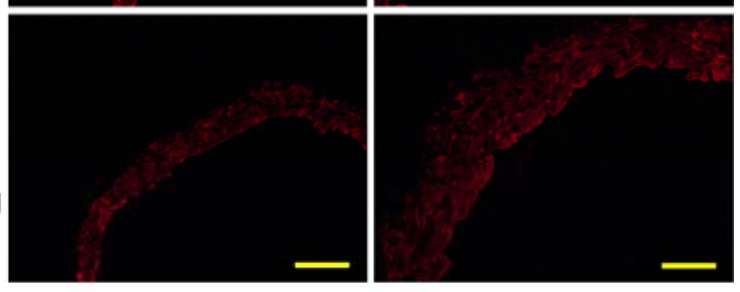

B

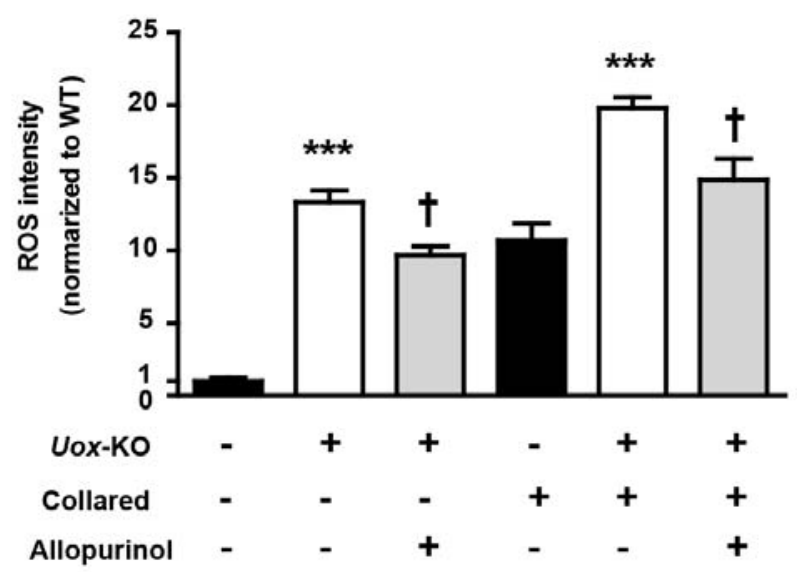


Figure 4.

A
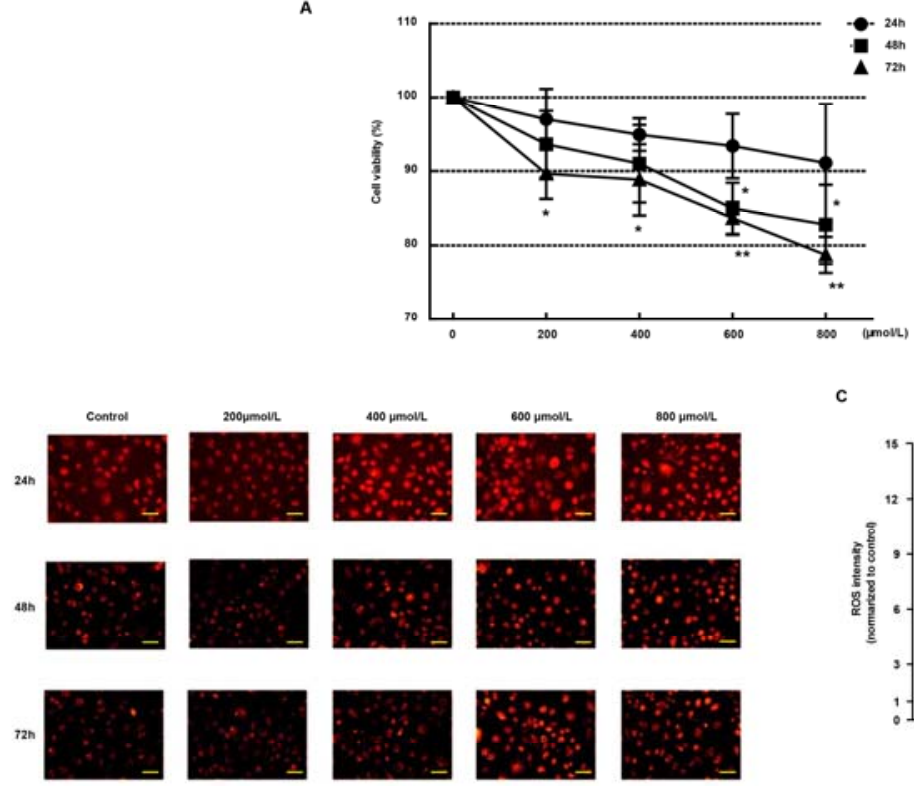

c
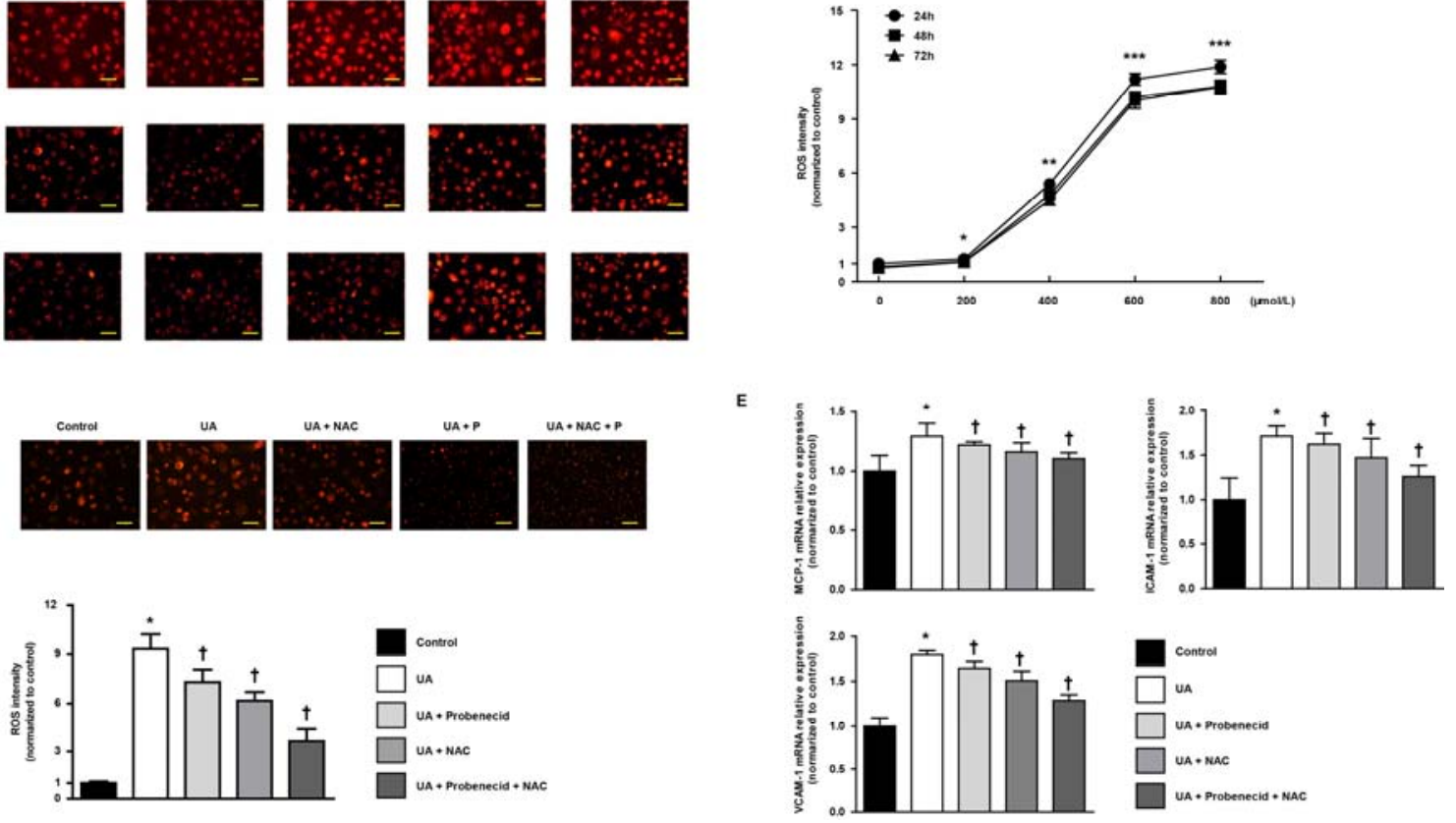
Table 1. Blood biochemistry in WT and Uox-KO mice

\begin{tabular}{|c|c|c|c|c|c|c|c|c|}
\hline & WT & KO & $P$ value & WT-collared & KO-collared & $P$ value & $\begin{array}{c}\text { KO-collared + } \\
\text { allopurinol }\end{array}$ & $\begin{array}{l}P \text { value (compared } \\
\text { to KO-collared) }\end{array}$ \\
\hline Uric acid $(\mu \mathrm{mol} / \mathrm{L})$ & $176.3 \pm 6.7$ & $563.9 \pm 16.9$ & $<0.001$ & $187.5 \pm 4.9$ & $573.0 \pm 16.4$ & $<0.001$ & $325.8 \pm 9.2$ & $<0.001$ \\
\hline Blood urea nitrogen $(\mathrm{mmol} / \mathrm{L})$ & $4.47 \pm 0.16$ & $10.04 \pm 0.35$ & $<0.001$ & $5.19 \pm 0.28$ & $10.42 \pm 0.42$ & $<0.001$ & $7.91 \pm 0.63$ & 0.005 \\
\hline Creatinine $(\mu \mathrm{mol} / \mathrm{L})$ & $16.53 \pm 1.67$ & $23.63 \pm 2.18$ & 0.04 & $15.36 \pm 1.83$ & $30.5 \pm 0.97$ & $<0.001$ & $21.77 \pm 2.47$ & 0.0047 \\
\hline Fasting glucose (mmol/L) & $6.0 \pm 0.2$ & $6.3 \pm 0.2$ & 0.25 & $6.1 \pm 0.2$ & $6.2 \pm 0.2$ & 0.71 & $6.4 \pm 0.3$ & 0.6 \\
\hline Total cholesterol (mmol/L) & $3.36 \pm 0.12$ & $3.67 \pm 0.13$ & 0.1 & $3.59 \pm 0.08$ & $3.85 \pm 0.12$ & 0.08 & $3.62 \pm 0.14$ & 0.23 \\
\hline Triglycerides (mmol/L) & $1.49 \pm 0.16$ & $1.13 \pm 0.03$ & 0.03 & $1.35 \pm 0.07$ & $1.23 \pm 0.03$ & 0.13 & $1.27 \pm 0.06$ & 0.62 \\
\hline HDL cholesterol (mmol/L) & $1.61 \pm 0.13$ & $1.74 \pm 0.05$ & 0.35 & $1.60 \pm 0.05$ & $1.80 \pm 0.08$ & 0.06 & $1.59 \pm 0.13$ & 0.21 \\
\hline LDL cholesterol (mmol/L) & $0.49 \pm 0.07$ & $0.55 \pm 0.04$ & 0.44 & $0.48 \pm 0.06$ & $0.55 \pm 0.06$ & 0.43 & $0.50 \pm 0.03$ & 0.44 \\
\hline
\end{tabular}

Biochemical indicators in WT and Uox-KO mice ( $n=8$ per group, males, 10-16 weeks of age). Data were shown as mean \pm SEM. 
Table 2. The results of transthoracic ultrasound

\begin{tabular}{|c|c|c|c|c|c|}
\hline & \multicolumn{2}{|c|}{ WT $(n=6)$} & \multicolumn{2}{|c|}{ KO $(n=6)$} & \multirow[b]{2}{*}{$\boldsymbol{P}$} \\
\hline & Mean & SEM & Mean & SEM & \\
\hline LVAW; d (mm) & 0.88 & 0.05 & 0.84 & 0.05 & 0.57 \\
\hline LVAW; s (mm) & 1.20 & 0.09 & 1.14 & 0.08 & 0.60 \\
\hline LVID; d (mm) & 3.43 & 0.07 & 3.72 & 0.13 & 0.08 \\
\hline LVID; s (mm) & 2.48 & 0.14 & 2.73 & 0.15 & 0.26 \\
\hline LVPW; d (mm) & 0.81 & 0.04 & 0.85 & 0.07 & 0.66 \\
\hline LVPW; s (mm) & 1.05 & 0.07 & 1.11 & 0.09 & 0.62 \\
\hline EF $(\%)$ & 54.38 & 4.40 & 52.04 & 4.93 & 0.73 \\
\hline FS (\%) & 27.77 & 2.81 & 26.52 & 3.01 & 0.77 \\
\hline LV Mass AW (mg) & 98.18 & 4.74 & 111.40 & 5.21 & 0.09 \\
\hline LV Mass AW (Corrected) (mg) & 78.54 & 3.79 & 89.12 & 4.16 & 0.09 \\
\hline LV Vol; d $(\mu \mathrm{L})$ & 48.47 & 2.43 & 59.50 & 4.91 & 0.07 \\
\hline LV Vol; s $(\mu \mathrm{L})$ & 22.61 & 3.11 & 28.59 & 3.89 & 0.26 \\
\hline Stroke volume $(\mu \mathrm{L})$ & 25.86 & 1.09 & 30.92 & 3.98 & 0.25 \\
\hline Cardiac output (mL/min) & 9.71 & 0.74 & 10.17 & 1.15 & 0.74 \\
\hline Heart rate (b.p.m.) & 375 & 22 & 342 & 35 & 0.50 \\
\hline
\end{tabular}

No significant difference was observed between male Uox-KO and WT counterparts $(n=6$, 10 weeks of age). LVAWd: end-diastolic left ventricular anterior wall thickness; LVAWs: endsystolic left ventricular anterior wall thickness; LVIDd: end-diastolic left ventricular inner diameter; LVIDs: end-systolic left ventricular inner diameter; LVPWd: end-dastolic left ventricular posterior wall thickness; LVPWs: end-systolic left ventricular posterior wall thickness; EF: ejection fraction; FS: fractional shortening; LV Mass AW: left ventricle mass; LV Vold: end-diastolic left ventricle volume; LV Vols: end-systolic left ventricle volume. 


\section{Supplementary Table 1}

Table 1. Primers of quantitative real-time RT-PCR

\begin{tabular}{|c|c|}
\hline Primers & $\left(\mathbf{5}^{\prime}\right.$-sequences-3 $\left.{ }^{\prime}\right)$ \\
\hline h-MCP-1_F & GATGCAATCAATGCCCCAGTC \\
\hline h-MCP-1_R & TCCTTGGCCACAATGGTCTTG \\
\hline h-ICAM-1_F & GGCTGGAGCTGTTTGAGAAC \\
\hline h-ICAM-1_R & TCACACTGACTGAGGCCTTG \\
\hline h-VCAM-1_F & TCCCTACCATTGAAGATACTGGAAA \\
\hline h-VCAM-1_R & GCTGACCAAGACGGTTGTATCTC \\
\hline h- $\beta$-actin_F & CGCAAAGACCTGTACGCCAAC \\
\hline h- $\beta$-actin_R & CACGGAGTACTTGCGCTCAGG \\
\hline
\end{tabular}

